\title{
Spinal Meninges
}

National Cancer Institute

\section{Source}

National Cancer Institute. Spinal Meninges. NCI Thesaurus. Code C12350.

Connective tissue membranes that surround and support the spinal cord and cauda

equina. They are continuous with cranial meninges, which surround and support the brain. 